OPEN ACCESS

Edited by:

Allison B. Reiss,

Winthrop University Hospital,

United States

Reviewed by:

Yi-Chung Lee,

Taipei Veterans General

Hospital, Taiwan

Raffaella Valenti,

University of Florence, Italy

*Correspondence:

Kazuo Washida

washida@ncvc.go.jp

Received: 02 December 2019

Accepted: 20 April 2020

Published: 14 May 2020

Citation:

Okada T, Washida K, Irie K, Saito S, Noguchi M, Tomita T, Koga M,

Toyoda K, Okazaki S, Koizumi T,

Mizuta I, Mizuno T and Ihara M (2020)

Prevalence and Atypical Clinical

Characteristics of NOTCH3 Mutations

Among Patients Admitted for Acute

Lacunar Infarctions.

Front. Aging Neurosci. 12:130.

doi: 10.3389/fnagi.2020.00130

\section{Prevalence and Atypical Clinical Characteristics of NOTCH3 Mutations Among Patients Admitted for Acute Lacunar Infarctions}

\author{
Takashi Okada ${ }^{1}$, Kazuo Washida ${ }^{1 *}$, Kenichi Irie ${ }^{1}$, Satoshi Saito ${ }^{1,2}$, Michio Noguchi $^{3}$, \\ Tsutomu Tomita ${ }^{3}$, Masatoshi Koga ${ }^{4}$, Kazunori Toyoda ${ }^{4}$, Shuhei Okazaki ${ }^{5}$, \\ Takashi Koizumi ${ }^{6}$, Ikuko Mizuta ${ }^{6}$, Toshiki Mizuno ${ }^{6}$ and Masafumi Ihara ${ }^{1}$ \\ ${ }^{1}$ Department of Neurology, National Cerebral and Cardiovascular Center, Osaka, Japan, ${ }^{2}$ Research Fellow of Japan Society \\ for the Promotion of Science, Tokyo, Japan, ${ }^{3}$ NCVC Biobank, National Cerebral and Cardiovascular Center, Osaka, Japan, \\ ${ }^{4}$ Department of Cerebrovascular Medicine, National Cerebral and Cardiovascular Center, Osaka, Japan, ${ }^{5}$ Department of \\ Neurology, Osaka University Graduate School of Medicine, Osaka, Japan, ${ }^{6}$ Department of Neurology, Graduate School of \\ Medical Science, Kyoto Prefectural University of Medicine, Kyoto, Japan
}

Objectives: Cerebral autosomal dominant arteriopathy with subcortical infarcts and leukoencephalopathy (CADASIL) is the most common hereditary small vessel disease, with reported frequencies of 2-5/100,000 individuals. Recently, it has been reported that some patients with $\mathrm{NOTCH} 3$ gene mutations show atypical clinical symptoms of CADASIL. Assuming that CADASIL is underdiagnosed in some cases of lacunar infarction, this study was designed to examine the prevalence of NOTCH3 gene mutations in the patients at highest risk who were admitted for lacunar infarctions.

Methods: From January 2011 to April 2018, 1,094 patients with lacunar infarctions were admitted to our hospital, of whom 31 patients without hypertension but with white matter disease (Fazekas scale 2 or 3) were selected and genetically analyzed for NOTCH3 gene mutations (Phase 1). Furthermore, 54 patients, who were 60 years or younger, were analyzed for NOTCH3 mutations (Phase 2). NOTCH3 exons 2-24, which encode the epidermal growth factor-like repeat domain of the NOTCH3 receptor, were analyzed for mutations by direct sequencing of genomic DNA.

Results: Three patients presented NOTCH3 p.R75P mutations: two in the Phase 1 and one in the Phase 2 cohort. Among patients aged 60 years or younger and those without hypertension but with moderate-to-severe white matter lesions, the carrier frequency of p.R75P was 3.5\% (3/85), which was significantly higher than that in the Japanese general population (4.7KJPN) (odds ratio [95\% Cl] $=58.2$ [11.6-292.5]). All three patients with NOTCH3 mutations had family histories of stroke, and the average patient age was 51.3 years. All three patients also showed white matter lesions in the external capsule but not in the temporal pole. The CADASIL and CADASIL scale-J scores of the three patients were 6, 17, 7 (mean, 10.0) and 13, 20, 10 (mean, 14.3), respectively.

Conclusion: Among patients hospitalized for lacunar infarctions, the p.R75P prevalence may be higher than previously estimated. The NOTCH3 p.R75P mutation may be 
underdiagnosed in patients with early-onset lacunar infarctions due to the atypical clinical and neuroimaging features of CADASIL. Early-onset, presence of family history of stroke, external capsule lesions, and absence of hypertension may help predict underlying $\mathrm{NOTCH3}$ mutations despite no temporal white matter lesions.

Keywords: CADASIL, CADASIL scale-J, NOTCH3, R75P mutation, lacunar infarction

\section{INTRODUCTION}

Cerebral autosomal dominant arteriopathy with subcortical infarcts and leukoencephalopathy (CADASIL) is an autosomal dominant disorder caused by mutations in the NOTCH3 gene in chromosome 19p13 (Tournier-Lasserve et al., 1993; Joutel et al., 1996) and is the most common hereditary small vessel disease, with clinical frequencies of 2-5/100,000 individuals (Joutel et al., 1997; Rutten et al., 2016). Patients with CADASIL have various clinical symptoms, such as lacunar infarction, migraines, progressive cognitive decline, and psychiatric problems (Chabriat et al., 2009), and the most specific imaging feature of patients with CADASIL are bilateral white matter hyperintensities (WMHs), especially WMHs of anterior temporal pole on magnetic resonance imaging (MRI) (Chabriat et al., 1995, 1998; Coulthard et al., 2000; Auer et al., 2001; Tomimoto et al., 2006; Bersano et al., 2018). However, some patients with NOTCH3 mutations do not show the typical clinical and imaging features of CADASIL (Kim et al., 2006; Mizuno et al., 2008; Sari et al., 2019). Furthermore, it has been recently clarified that the potential prevalence of CADASIL may be higher than previously estimated (Rutten et al., 2016). Assuming that NOTCH3 gene mutations may be involved in some cases of lacunar infarction, a representative small vessel disease, this study was designed to investigate the prevalence and clinical characteristics of $\mathrm{NOTCH} 3$ gene mutations in the patients at highest risk who were admitted for lacunar infarction, using a whole sequence analysis of NOTCH3 genes.

\section{METHODS}

\section{Study Design and Participants}

This single-center cross-sectional study was performed at the National Cerebral and Cardiovascular Center (NCVC) of Osaka, Japan, and conducted in accordance with Declaration of Helsinki standards and after approval by the local NCVC ethical committee (M29-117-3). All participants signed a comprehensive NCVC biobank consent form.

The subjects of this study were patients with lacunar infarction with hyperintense signal on MRI Diffusion-weighted imaging (DWI).

Lacunar infarction was divided into two types based on the diameter of the occluded vessels: lacunar infarction of $20 \mathrm{~mm}$ or less in diameter and penetrating artery occlusion stroke exceeding $20 \mathrm{~mm}$ in diameter. Lacunar infarction was defined as lesions of $20 \mathrm{~mm}$ or less in the brainstem or subcortex in the territory of a penetrating artery. In principle, there was no major artery stenosis and no embolic sources. Penetrating artery occlusion stroke was defined as lesions exceeding $20 \mathrm{~mm}$ in the brainstem or subcortex in the territory of a penetrating artery. In principle, there was no major artery stenosis and no embolic sources.

We defined the inclusion criteria for cohort 1 and 2 to evaluate the frequency of CADASIL in the patients with the highest risk because genetic testing of all 1,094 patients is impractical in terms of cost and time.

In this study, therefore, hypertension and aging were used as exclusion criteria for patient selection because these are the two strongest risk factors for lacunar infarction (Veglio et al., 2009; Regenhardt et al., 2018; Kalaria and Hase, 2019). In addition, white matter lesions were used as inclusion criteria for patient selection because white matter lesions were the most representative imaging finding of CADASIL (Chabriat et al., 1998; Koizumi et al., 2019).

Among patients with acute lacunar infarctions, patients without hypertension but with white matter disease (Fazekas scale 2 or 3) were selected and genetically analyzed for NOTCH3 gene mutations, in order to strictly target patients with lacunar infarction of $20 \mathrm{~mm}$ or less in diameter (Phase 1). Furthermore, patients with acute lacunar infarctions or penetrating artery occlusion stroke aged 60 years old or younger were genetically analyzed for $\mathrm{NOTCH} 3$ gene mutations in order to broadly target patients with lacunar infarctions or penetrating artery occlusion stroke (Phase 2).

Patients who satisfied the following criteria were included in this study:

- Phase 1 cohort: (1) patients who deposited samples in the NCVC biobank from January 2011 to April 2018, (2) patients with lacunar infarction $(20 \mathrm{~mm}$ or less in diameter) without a past history of hypertension, and (3) patients with deep white matter hyperintensity (DWMH) or periventricular hyperintensity (PVH) of grade 2 or higher on the Fazekas scale (Fazekas et al., 1991).

- Phase 2 cohort: (1) patients who deposited samples in NCVC biobank from January 2011 to April 2018 and (2) patients with acute lacunar infarctions $(20 \mathrm{~mm}$ or less in diameter) or penetrating artery occlusion stroke (exceeding $20 \mathrm{~mm}$ in diameter) who experienced their first stroke at the age of 60 years or younger.

- Lacunar infarction, penetrating artery occlusion stroke, and white matter lesions were diagnosed based on MRI. Patients who had already been diagnosed with CADASIL were excluded.

- A patient selection flow diagram is detailed in Figures 1, 2.

\section{Clinical Assessment}

We collected clinical information that included data on clinical background (age at onset, sex, family history, and vascular risk factors), neurological symptoms, and MRI 


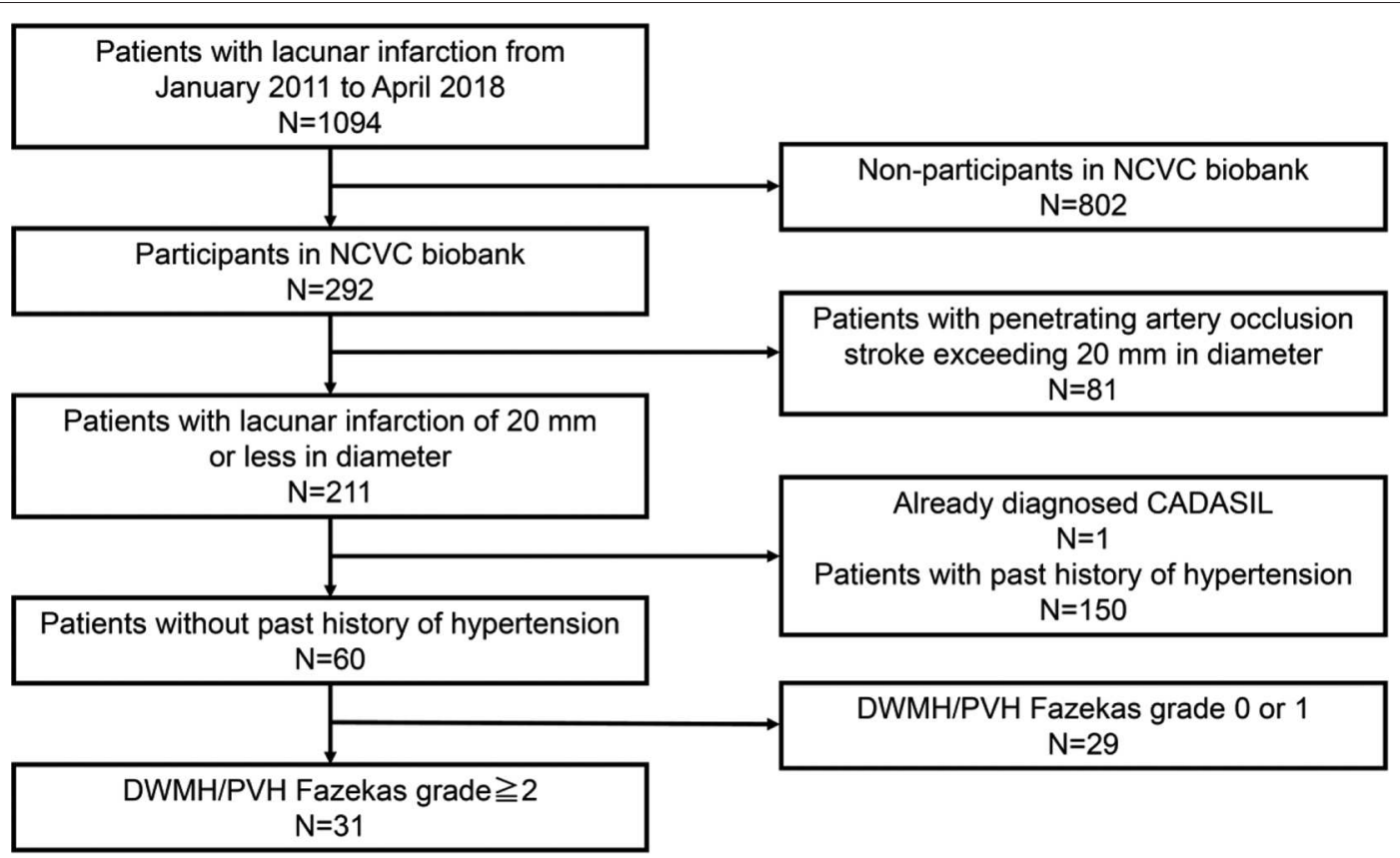

FIGURE 1 | Patient selection flow diagram for the phase 1 cohort. NCVC, National Cerebral and Cardiovascular Center; CADASIL, Cerebral autosomal dominant arteriopathy with subcortical infarcts and leukoencephalopathy; DWMH, deep white matter hyperintensity; PVH, periventricular hyperintensity.

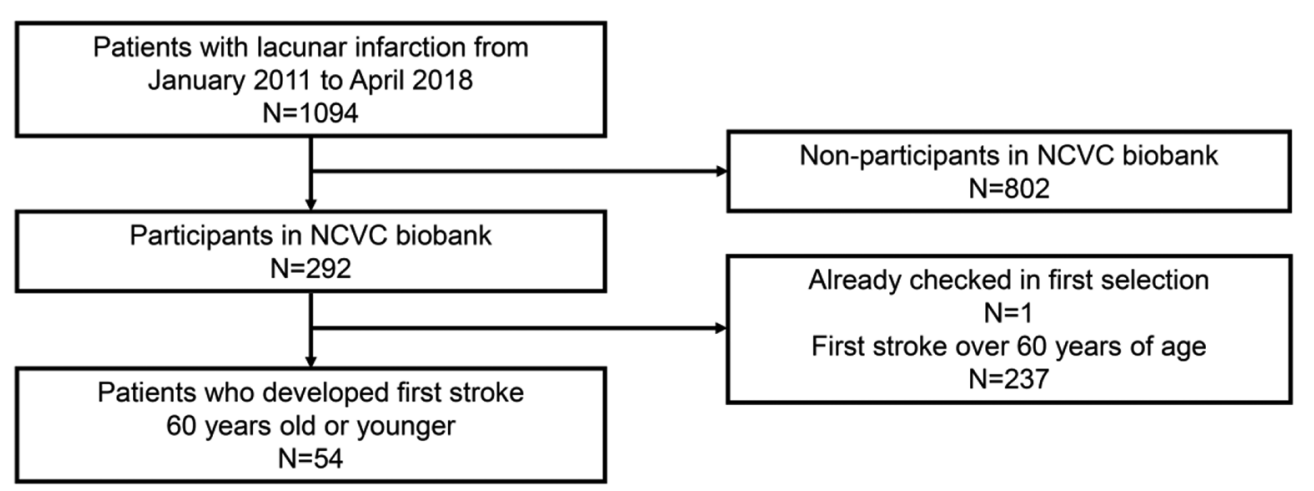

FIGURE 2 | Patient selection flow diagram for the phase 2 cohort. NCVC, National cerebral and cardiovascular center.

findings. The neurological symptoms that were evaluated included stroke, migraine, motor palsy, sensory disturbance, dizziness, pseudobulbar palsy, seizure, mood disturbance, and cognitive impairment.

Lacunar infarctions were diagnosed according to the Trial of Org 10172 Acute Stroke Treatment Criteria (Adams et al., 1993). Hypertension was defined as a blood pressure of at least $140 / 90 \mathrm{mmHg}$ on two separate measurements or the use of antihypertensive drugs. Diabetes mellitus was defined as a fasting blood glucose level of at least $126 \mathrm{mg} / \mathrm{dL}, \mathrm{HbA} 1 \mathrm{c} 6.5 \%$ or higher, or the use of antidiabetic medications. Dyslipidemia was defined as a fasting serum low-density lipoprotein cholesterol level of at least $140 \mathrm{mg} / \mathrm{dL}$ or use of cholesterol-lowering therapy. Smoking status was determined by self-reporting as either a smoker (current or ex-smoker) or a nonsmoker. A family history of stroke was determined based on combined responses of the patient, sibling, and child to questions.

\section{Magnetic Resonance Imaging Protocol}

Participants underwent imaging of the brain with a 3.0 Tesla MRI (Magnetom Verio or Spectra; Siemens Medical Solutions, Erlangen, Germany) scanner. A standardized clinical protocol was employed that included DWI and apparent diffusion coefficient (ADC), fluid-attenuated inversion recovery (FLAIR), $\mathrm{T} 2$ star weighted imaging (T2* WI), and MR angiography.

\section{Application of the CADASIL Scale and CADASIL Scale-J}

The CADASIL scale of Pescini et al. (2012) is a simple scale that can be applied in a clinical setting as a screening tool for 
predicting a genetic diagnosis of CADASIL. The scale involves the additive score of 12 items (ranging from 0 to 25), whose cut-off value is 15 . Result categories of the CADASIL scale were determined as positive $(\geq 15)$ or negative $(<15)$.

The CADASIL scale-J of Koizumi et al. (2019) is a modified, Japanese version of the CADASIL scale, with a score range from 0 to 25 and cut-off value of 16 . This version uses eight items: hypertension, diabetes, young onset ( $\leq 50$ years old), pseudobulbar palsy, stroke/TIA, family history, subcortical infarction, and temporal pole lesion.

\section{Genetic Examination}

Genetic testing of NOTCH3 was performed as described previously (Mizuta et al., 2017). In brief, NOTCH3 exons 2-24, which encode the epidermal growth factor-like repeat (EGFr) domain of the NOTCH3 receptor, were analyzed for mutations by direct sequencing of genomic DNA that was extracted from the peripheral blood. The sequence data were analyzed with SEQUENCHER (Gene Codes, HITACHI) to screen for mutations. Nucleotide substitutions were confirmed by restriction fragment length polymorphism analysis. We concluded that the variation was pathogenic when it was previously reported as pathogenic and/or when it resulted in a cysteine-related missense mutation in one EGFr.

\section{RESULTS}

\section{Baseline Demographics and Participants}

During the study period, 1,094 patients with lacunar infarctions were admitted to the hospital (mean age \pm standard deviation (SD), $72.0 \pm 11.0 ; 61.0 \%$ men). In total, 292 of 1,094 patients participated in the NCVC biobank after providing written informed consent.

Of the overall cohort, 31 patients without hypertension but with white matter disease (Fazekas scale 2 or 3 ) were selected and genetically analyzed for $\mathrm{NOTCH} 3$ gene mutations in the phase 1 cohort (mean age $\pm S D, 77.4 \pm 9.611 .0 ; 76.5 \%$ men) (Figure 1). The demographic data of the 31 patients in the phase 1 cohort are shown in Table 1.

TABLE 1 | Demographic data of the 31 patients in the Phase 1 cohort.

\begin{tabular}{lc}
\hline Demographics in $\mathbf{3 1}$ patients in Phase 1 cohort \\
\hline Age, years (SD) \\
Sex \\
$\quad$ Men, $n(\%)$ & $77.4(9.6)$ \\
$\quad$ Women, $n(\%)$ & $23(76.5 \%)$ \\
Median NIHSS at baseline & $8(23.5 \%)$ \\
HTN, $n(\%)$ & $3($ IQR $2-4)$ \\
DL, $n(\%)$ & $0(0 \%)$ \\
DM, $n(\%)$ & $17(82.4 \%)$ \\
Smoking, $n(\%)$ & $12(40 \%)$ \\
\hline
\end{tabular}

$S D$, Standard deviation; NIHSS, national institute of health stroke scale; IQR, interquartile range; HTN, hypertension; DL, dyslipidemia; DM, diabetes mellitus.
Furthermore, 54 patients, who were 60 years old or younger, were genetically analyzed for NOTCH3 gene mutations in the phase 2 cohort (mean age $\pm S D, 53.0 \pm 5.7 ; 77.8 \%$ men) (Figure 2). The demographic data of the 54 patients in the phase 2 cohort are shown in Table 2.

\section{Prevalence of NOTCH3 Mutations Among Patients With Lacunar Infarctions}

Three patients had NOTCH3 p.R75P mutations (ClinVar accession number: VCV000632306.1): two in the Phase 1 cohort (cases 1 and 2) and one in the phase 2 cohort (case 3 ). Among patients aged 60 years or younger or patients without hypertension with moderate-to-severe white matter lesions, the carrier frequency of p.R75P was 3.5\% (3/85), which was significantly higher than that in the Japanese general population $(4.7 \mathrm{KJPN})(n=4773)$ (odds ratio $[95 \% \mathrm{CI}]=58.2$ [11.6-292.5]) (Table 3). The p.R75P carrier frequency in biobank patients with lacunar infarction $(1.0 \%, 3 / 292)$ was also significantly higher than that in $4.7 \mathrm{KJPN}$ (odds ratio [95\% CI] $=16.5$ [3.3-82.1]).

The three patients with p.R75P mutations had no blood causality each.

\section{Clinical Characteristics of Patients With p.R75P Mutations}

The clinical characteristics of patients with p.R75P mutations are shown in Table 4. The average patient age was 51.3 years. All patients had dyslipidemia and family histories of stroke; however, only the patient in case 2 showed cognitive impairment while the patient in case 3 had hypertension. No history of diabetes mellitus or cigarette smoking was found. Furthermore, the patients in case 1 and 3 had symptoms of dysarthria and dysesthesia but did not show typical clinical characteristics of CADASIL such as migraine and temporal pole lesions. The patient in case 2 showed relatively typical clinical symptoms, such as migraines and cognitive impairment.

\section{Brain MRI Characteristics of Patients With p.R75P Mutations}

All three patients with p.R75P mutations had no temporal pole lesions (Figures 3A,E,I), while hyperintensity lesions were

TABLE 2 | Demographic data of the 54 patients in the Phase 2 cohort.

\begin{tabular}{ll}
\hline Demographics in $\mathbf{5 4}$ patients in Phase 2 cohort & \\
\hline Age, years (SD) & $53.0(5.7)$ \\
Sex & \\
$\quad$ Men, $n$ (\%) & $42(77.8 \%)$ \\
$\quad$ Women, $n$ (\%) & $12(22.2 \%)$ \\
Median NIHSS at baseline & $3($ IQR $2-4)$ \\
HTN, $n(\%)$ & $45(83.3 \%)$ \\
DL, $n(\%)$ & $39(72.2 \%)$ \\
DM, $n(\%)$ & $22(40.7 \%)$ \\
Smoking, $n(\%)$ & $33(61.1 \%)$
\end{tabular}

$S D$, Standard deviation; NIHSS, national institute of health stroke scale; IQR, interquartile range; HTN, hypertension; DL, dyslipidemia; DM, diabetes mellitus. 
TABLE 3 | Frequency of NOTCH3 p.R75P mutations in patients and controls.

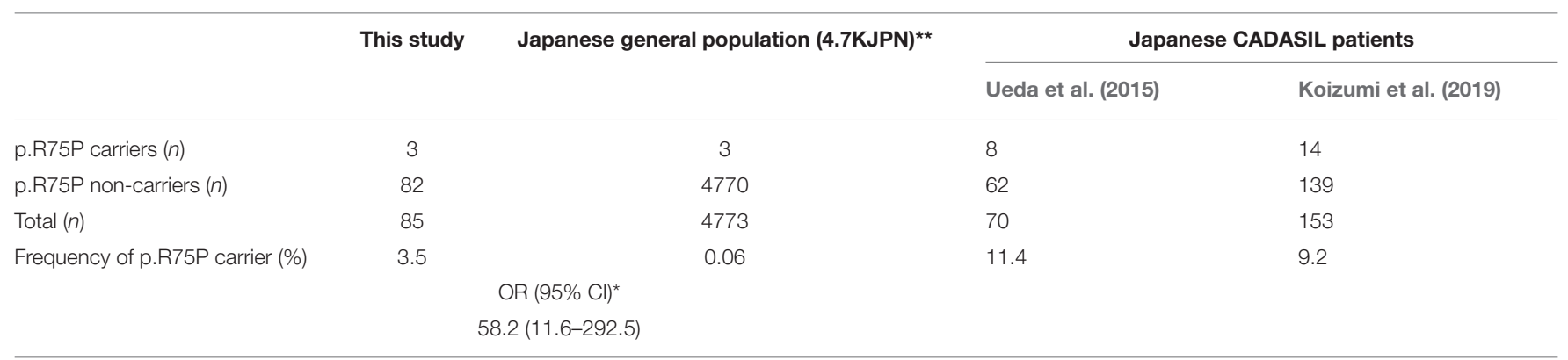

*Internet calculator (http://www.hutchon. net/ConfidOR.htm) was used.

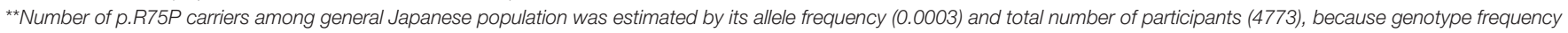
is not freely available on 4.7KJPN yet.

$\mathrm{OR}$, Odds ratio; $\mathrm{Cl}$, confidence interval.

noticeable at the external capsule (Figures 3B,F,J), and the white matter lesions that were evaluated by the Fazekas scale were of grades 2-3 (Figures 3C,D,G,H,K,L). The patients in cases 1 and 2 exhibited several cerebral microbleeds, while the patient in case 3 had no cerebral microbleeds on T2*WI. MR angiography showed no arterial stenoses in all three patients with CADASIL.

\section{CADASIL Scale and CADASIL Scale-J Scores}

The CADASIL and CADASIL scale-J scores of the three patients were $6,17,7$ (mean, 10.0) and 13, 20, 10 (mean, 14.3), respectively (Table 4). The CADASIL scale-J score was higher than that of the CADASIL scale, although both scale scores were below their respective cut-off values (CADASIL scale: $\geq 15 / 25$ ); CADASIL scale-J: $\geq 16 / 25$ ).

\section{DISCUSSION}

In this study, the frequency of NOTCH3 mutations was investigated for the first time in a Japanese lacunar stroke cohort; three patients had cysteine-sparing NOTCH3 p.R75P mutations: two in the phase 1 cohort and one in the phase 2 cohort. The carrier frequency of p.R75P in this study was $3.5 \%(3 / 85)$, which was significantly higher than that in the Japanese general population $(4.7 \mathrm{KJPN}$ ) (odds ratio [95\% CI] $=58.2$ [11.6-292.5]). Furthermore, all three patients with NOTCH3 mutations had family histories of stroke and showed hyperintensity lesions at external capsules and moderate-tosevere white matter lesions. However, these patients did not show typical CADASIL clinical and imaging features, such as temporal pole lesions. The CADASIL scale-J score was higher than that of the CADASIL scale.

CADASIL is the most common hereditary small vessel disease, and the estimated prevalence is 2-5/100,000 individuals in clinical practice (Joutel et al., 1997). The most frequent clinical symptoms are ischemic events (59\%) and psychiatric disturbances (48\%) (Bianchi et al., 2015). Migraine with aura and subcortical vascular cognitive impairment, which are associated with pseudobulbar palsy and urinary incontinence, are also characteristic symptoms of CADASIL (Chabriat et al., 2009). However, it has recently been reported that a subset of patients with NOTCH3 gene mutations show atypical clinical symptoms of CADASIL, such as absence of temporal pole lesions (Ueda et al., 2015), elderly onset (Watanabe et al., 2012), and cerebellar atrophy (Sari et al., 2019). Furthermore, the exome aggregation consortium (ExAC) database study identified 206 EGFr cysteine-altering NOTCH3 mutations in the ExAC, with an overall prevalence of 3.4/1,000 individuals (Rutten et al., 2016). The prevalence of CADASIL may be higher than previously thought; in fact, Dong Y et al. reported that one patient with a p.C697T mutation was found among 218 consecutive patients with lacunar infarction in the United Kingdom (Dong et al., 2003). Furthermore, Choi et al. reported that six patients with p.R544C mutations were found among 151 consecutive Korean patients with acute ischemic stroke (Choi et al., 2013), four of whom presented with large artery atherosclerosis and two with lacunar infarction. Our findings echo recent findings in Taiwan and the United Kingdom. Lee et al. reported that the NOTCH3 p.R544C mutation was present in a significant number of individuals in Taiwan, including 60 of 7,038 healthy controls (0.9\%), 17 of 800 patients with ischemic stroke $(2.1 \%)$, and 16 of 245 patients with small vessel occlusion stroke (6.5\%) from the Taiwan Biobank and that the other two cysteine-altering mutations (p.C853Y, and p.C884Y) were rarely detected (Lee et al., 2020). Furthermore, from the UK DNA Lacunar Stoke Study, Tan et al. identified single gene mutations in 14 patients (eight cysteine-altering NOTCH3 variants in 11 patients, two HTRA1 variants in two patients, and one missense COL4A1 variant in one patient) among 950 patients with younger-onset apparently sporadic small vessel disease stroke using a targeted sequencing panel (14 of 950; 1.5\%; Tan et al., 2019). These results strongly support our findings, which indicate that NOTCH3 gene mutations may be involved in some cases of lacunar infarction, which is a representative small vessel disease. CADASIL is probably underdiagnosed in the wider stroke population.

In this study, all three patients with NOTCH3 p.R75P mutations were relatively young and had family histories of stroke. Furthermore, all three patients showed hyperintensity lesions at external capsules and moderate-to-severe white matter lesions, but did not show temporal pole lesions. Temporal pole 


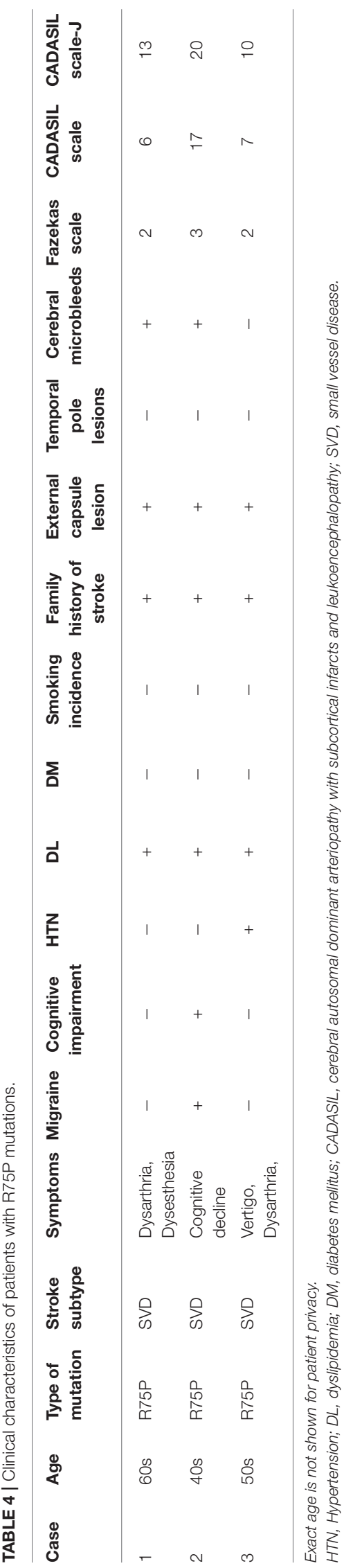

white matter lesions on MRI is one of the most characteristic imaging features of patients with CADASIL (Singhal et al., 2005; Yamamoto et al., 2009); however, some patients with CADASIL do not show temporal pole lesions. Abnormal temporal pole white matter lesions are less common in Asian patients than among Caucasians (Wang et al., 2011), and the prevalence of temporal pole involvement is only between $33.8-71 \%$ in the Japanese population (Ueda et al., 2015). In particular, cysteinesparing NOTCH3 missense mutations, such as p.R75P (Kim et al., 2006), p.R61W (Brass et al., 2009), p.D80G (Wollenweber et al., 2015), and p.R213K (Santa et al., 2003) are associated with atypical MRI findings with less anterior temporal lobe involvement (Matsushima et al., 2017; Muino et al., 2017). In a prior study of patients with CADASIL, $91 \%$ of cysteinesparing $\mathrm{NOTCH} 3$ mutations did not have anterior temporal pole involvement (Muino et al., 2017). Furthermore, the cysteine at codon 76 could be related to fixation of the double hairpin in the NOTCH3 EGFr, and the substitution of the basic arginine for a proline at codon 75 could induce a partial three-dimensional conformational change in the EGFr that leads to less involvement of the anterior temporal lobe (Mizuno et al., 2008). The precise mechanisms by which cysteine-sparing $\mathrm{NOTCH} 3$ missense mutations induce CADASIL remains to be elucidated. We previously showed that temporal pole changes partially reflect dilated perivascular spaces (Yamamoto et al., 2009). Therefore, the p.R75P mutation may be less likely to dilate perivascular spaces than the cysteine-substitution mutations of NOTCH3. Cysteine-sparing mutations should be further studied to confirm their pathological role in CADASIL (Rutten et al., 2014; Coupland et al., 2018). Interestingly, CADASIL patients with NOTCH3 p.R75P were frequently found throughout Japan. The allele frequency of p.R75P in the general population is 0.0003 , according to the largest Japanese whole genome reference panel 4.7KJPN (https://jmorp.megabank.tohoku.ac.jp/202001/variants [accessed 25 March 2020], in the Japanese Multi Omics Reference Panel [jMorp], Tohoku Medical Megabank Organization). It is of note that the carrier frequency of p.R75P in this study was $3.5 \%$ (3/85), which was significantly higher than that in $4.7 \mathrm{KJPN}$ (odds ratio [95\% CI] $=58.2[11.6-292.5]$ ). The p.R75P carrier frequency in biobank patients with lacunar infarction $(1.0 \%, 3 / 291)$ was also significantly higher than that in $4.7 \mathrm{KJPN}$ (odds ratio $[95 \% \mathrm{CI}]=16.5$ [3.3-82.1]). Our finding is compatible with the high frequency of p.R75P (9.211.4\%) among Japanese CADASIL patients (Ueda et al., 2015; Koizumi et al., 2019). Mukai et al. analyzed the genotypephenotype correlation based on the three most common mutations in Japanese CADASIL patients: the cysteine-sparing NOTCH3 mutation p.R75P and cysteine-altering NOTCH3 mutations p.R141C and p.R182C. p.R141C showed the most typical CADASIL phenotypes while p.R75P showed mild and atypical phenotypes, with a low frequency of temporal pole lesions, high frequency of hypertension, and low frequency of stroke/transient ischemic attack (TIA). Phenotypes of p.R182C were similar to those of p.R141C, except for a lower frequency of stroke/TIA. Initial symptoms of the 14 probands with p.R75P were none (four of 14; 28.6\%), cognitive impairment (three of 14; $21.4 \%$ ), depression (one of $14,7.1 \%$ ), and stroke/TIA (six 


\section{Case 1}
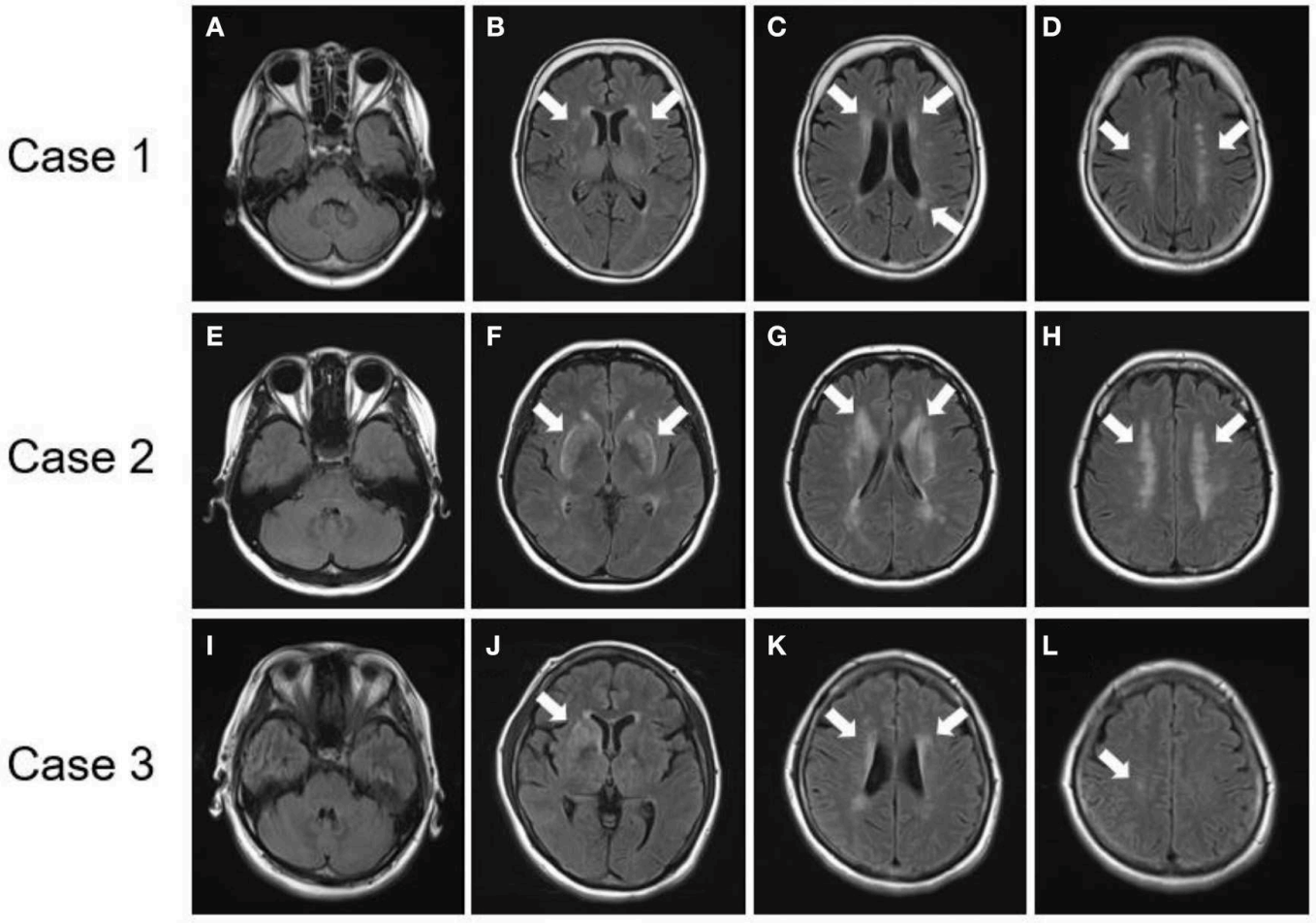

FIGURE 3 | Brain MRI findings of patients with NOTCH3 mutation. (A-D) FLAIR images of a patient with a NOTCH3 p.R75P mutation (Case 1). (E-H) FLAIR images of a patient with a NOTCH3 p.R75P mutation (Case 2). (I-L) FLAIR images of a patient with a NOTCH3 p.R75P mutation (Case 3). (A,E,I) No patients presented lesions on their temporal pole lobes. (B,F,J) Arrows indicate hyperintensity lesions at external capsules. (C,D,G,H,K,L) Arrows indicate periventricular hyperintensity.

of 14; 42.9\%; Mukai et al., 2020). p.R75P presents as clinical symptoms that resemble sporadic lacunar infarcts, and head MRI often shows external capsule lesions and white matter lesions without temporal pole lesions (Kim et al., 2014; Ueda et al., 2015). Therefore, cysteine-sparing NOTCH3 p.R75P mutation may be underdiagnosed in early-onset lacunar infarctions in daily clinical practice. Lacunar infarctions and white matter lesions of the external capsule, along with family history of stroke, can be prognostic factors that suggest the need for further genetic testing for possible underlying NOTCH3 mutations.

NOTCH3 genetic testing remains the diagnostic gold standard, and a clinical screening tool is eagerly desired because genetic testing is costly and time-consuming. Pescini et al. proposed the CADASIL scale, a screening tool that can be used to select patients for NOTCH3 gene analysis (Pescini et al., 2012). The CADASIL scale showed high sensitivity in European studies; however, low sensitivity was reported in Chinese patients, possibly due to ethnic differences in clinical manifestations (Liu et al., 2015). Recently, Koizumi et al. developed the CADASIL scale-J, which can effectively discriminate between patients with CADASIL and patients with NOTCH3-negative CADASIL-like symptoms, among Japanese patients. The CADASIL scale-J exhibited a higher diagnostic accuracy than that of the CADASIL scale (Koizumi et al., 2019). The CADASIL scale-J was also well validated in 69 consecutive patients who underwent genetic testing. As a result, the sensitivity and specificity of the CADASIL scale-J were 78.9 and $85.7 \%$, respectively (Koizumi et al., 2019).
Furthermore, the CADASIL scale and the CADASIL scale-J scores of the patients with p.R75P were 6, 7, 17, and 10, 13, 20, respectively, in this study. While the CADASIL scale-J score was higher than that of the CADASIL scale, both scores were below their respective cut-off values (CADASIL scale: $\geq 15 / 25$, CADASIL scale-J: $\geq 16 / 25$ ). The low average scores in patients with p.R75P mutations may be due to a lower frequency of temporal pole lesions and older age onset compared with other CADASIL subtypes, such as cysteine-altering NOTCH3 mutations (Ueda et al., 2015). The p.R75P mutation, which involves an arginine residue rather than a cysteine, is related to less frequent involvement of the anterior temporal area, thus hampering the clinical utility of CADASIL screening tools. It has been also reported that there is no single pathogenomic clinical or neuroimaging finding that can distinguish patients with CADASIL from those with sporadic stroke (Pantoni et al., 2010). Therefore, the present study supports the notion that clinicians should exercise caution concerning the possibility that atypical patients with $\mathrm{NOTCH} 3$ mutations, such as p.R75P, may be unrecognized among patients with lacunar infarctions due to the atypical clinical and neuroimaging features of CADASIL.

From management and therapeutic perspectives, no definitive treatment has been established for CADASIL progression (Bersano et al., 2017). However, the early diagnosis and treatment for CADASIL, including the strict management of modifiable vascular risk factors, can improve patient prognosis. There are several reports about management options for CADASIL, and 
active smoking should be avoided, because it is independently associated with an earlier age of CADASIL onset and increased risk of stroke (Singhal et al., 2004; Chabriat et al., 2016). Additionally, the adequate control of homocysteine levels could decrease the risk of migraines (Singhal et al., 2004). Hypertension is also associated with an increased risk of stroke (Adib-Samii et al., 2010), cerebral microbleeds (Lee et al., 2017), intracerebral hemorrhage, and brain volume changes (Peters et al., 2006), as well as clinical progression. Other modifiable risk factors, such as diabetes mellitus (Viswanathan et al., 2006), alcohol abuse, and obesity, should also be controlled to prevent disease progression (Bersano et al., 2017). Furthermore, the administration of lomerizine, a diphenylmethylpiperazine $\mathrm{Ca}^{2+}$ channel blocker, could improve cognitive impairment and cerebral hypoperfusion in patients with CADASIL (Mizuno et al., 2009). Thus, the early detection of underdiagnosed CADASIL in patients who are initially admitted for lacunar infarctions should be carefully monitored to improve prognosis.

There are several limitations associated with the present study that warrant mentioning. First, this study is based on a relatively small number of patient because the genetic examination of all 1,094 patients was impractical in terms of cost and time. Patients aged 60-70 years and/or with white matter disease could be good targets for genetic testing (Tan et al., 2019; Lee et al., $2020)$. Age (60 years or younger), hypertension, and white matter lesions were used as criteria for patient selection in this study but these cohort selection criteria could be seen as arbitrary. Other characteristic findings of CADASIL, such as migraine, psychiatric symptoms, dementia, and family history of stroke could also be useful criteria for patient selection. More data are necessary to confirm the validity of our findings and their generalizability across other patient populations in the future. Second, this study was conducted at a single-center that was specialized for stroke and cardiovascular disease. Thus, selection biases may have led to an insufficient estimation of the frequency of NOTCH3 gene mutations across more general populations. Further multi-center studies are needed to confirm the precise association of NOTCH3 gene mutations with lacunar infarctions. Third, we only analyzed patients with lacunar infarctions in this study, and this could cause a bias in examining the exact prevalence of $\mathrm{NOTCH} 3$ mutations among patients with overall subtypes of ischemic stroke, including large artery atherosclerosis, cardioembolism, and ischemic stroke of undetermined etiology. Further analyses including overall subtypes of ischemic stroke are necessary to confirm the exact prevalence of $\mathrm{NOTCH} 3$ mutations in the future.

\section{REFERENCES}

Adams, H. P. Jr., Bendixen, B. H., Kappelle, L. J., Biller, J., Love, B. B., Gordon, D. L., et al. (1993). Classification of subtype of acute ischemic stroke. Definitions for use in a multicenter clinical trial. Toast. Trial of org 10172 in acute stroke treatment. Stroke 24, 35-41. doi: 10.1161/01.STR. 24.1.35

Adib-Samii, P., Brice, G., Martin, R. J., and Markus, H. S. (2010). Clinical spectrum of cadasil and the effect of cardiovascular risk factors on phenotype:

\section{CONCLUSION}

Among patients hospitalized for lacunar infarction, the carrier frequency of p.R75P may be higher than previously estimated. Furthermore, the NOTCH3 p.R75P mutation may be underdiagnosed in early-onset lacunar infarctions due to the atypical clinical and neuroimaging features of CADASIL. Early-onset, non-hypertensive lacunar infarcts and white matter lesions of the external capsule, and a family history of stroke are candidate factors that suggest the need for further genetic testing for underlying NOTCH3 mutations.

\section{DATA AVAILABILITY STATEMENT}

The genetic data generated in this study can be obtained on NCBI ClinVar, https://www.ncbi.nlm.nih.gov/clinvar/, under accession no: VCV000632306.1.

\section{ETHICS STATEMENT}

The studies involving human participants were reviewed and approved by Local ethics committee of NCVC. The patients/participants provided their written informed consent to participate in this study.

\section{AUTHOR CONTRIBUTIONS}

TO and KW: drafted the manuscript, designed the study, and acquired and analyzed the data. KI, SS, and MN: acquired and interpreted the data. SO, TT, MK, KT, and MI: revised the manuscript, interpreted the data, and supervised the study. TK, IM, and TM: performed the NOTCH3 genetic examinations.

\section{FUNDING}

This work was supported by the Grant-in-Aid for Scientific Research of Japan on Priority Areas from the Japanese Ministry of Education, Science and Culture: 19K21321 (KW).

\section{ACKNOWLEDGMENTS}

We would like to thank patients and families who participated in this study. This research was performed using samples acquired from the National Center Biobank Network (NCBN)/ NCVC Biobank resource. For further details, please see http://www. ncbiobank.org/ and http://www.ncvc.go.jp/biobank/. 
Bersano, A., Bedini, G., Oskam, J., Mariotti, C., Taroni, F., Baratta, S., et al. (2017). Cadasil: Treatment and management options. Curr. Treat. Options Neurol. 19:31. doi: 10.1007/s11940-017-0468-Z

Bianchi, S., Zicari, E., Carluccio, A., Di Donato, I., Pescini, F., Nannucci, S., et al. (2015). Cadasil in central italy: a retrospective clinical and genetic study in 229 patients. J. Neurol. 262, 134-141. doi: 10.1007/s00415-014-7533-2

Brass, S. D., Smith, E. E., Arboleda-Velasquez, J. F., Copen, W. A., and Frosch, M. P. (2009). Case records of the massachusetts general hospital. Case 12-2009. A 46-year-old man with migraine, aphasia, and hemiparesis and similarly affected family members. N. Engl. J. Med. 360, 1656-1665. doi: 10.1056/NEJMcpc0810839

Chabriat, H., Herve, D., Duering, M., Godin, O., Jouvent, E., Opherk, C., et al. (2016). Predictors of clinical worsening in cerebral autosomal dominant arteriopathy with subcortical infarcts and leukoencephalopathy: Prospective cohort study. Stroke 47, 4-11. doi: 10.1161/STROKEAHA.115.010696

Chabriat, H., Joutel, A., Dichgans, M., Tournier-Lasserve, E., and Bousser, M. G. (2009). The lancet. Neurology 8, 643-653. doi: 10.1016/S1474-4422(09)70127-9

Chabriat, H., Levy, C., Taillia, H., Iba-Zizen, M. T., Vahedi, K., Joutel, A., et al. (1998). Patterns of mri lesions in cadasil. Neurology 51, 452-457. doi: 10.1212/WNL.51.2.452

Chabriat, H., Vahedi, K., Iba-Zizen, M. T., Joutel, A., Nibbio, A., Nagy, T. G., et al. (1995). Clinical spectrum of cadasil: A study of 7 families. Cerebral autosomal dominant arteriopathy with subcortical infarcts and leukoencephalopathy. Lancet 346, 934-939. doi: 10.1016/S0140-6736(95)91557-5

Choi, J. C., Lee, K. H., Song, S. K., Lee, J. S., Kang, S. Y., and Kang, J. H. (2013). Screening for notch 3 gene mutations among 151 consecutive korean patients with acute ischemic stroke. J. Stroke Cerebrovasc. Dis. 22, 608-614. doi: 10.1016/j.jstrokecerebrovasdis.2011.10.013

Coulthard, A., Blank, S. C., Bushby, K., Kalaria, R. N., and Burn, D. J. (2000). Distribution of cranial mri abnormalities in patients with symptomatic and subclinical ca.dasil. Br. J. Radiol. 73, 256-265. doi: 10.1259/bjr.73.867.10817040

Coupland, K., Lendahl, U., and Karlstrom, H. (2018). Role of notch3 mutations in the cerebral small vessel disease cerebral autosomal dominant arteriopathy with subcortical infarcts and leukoencephalopathy. Stroke 49, 2793-2800. doi: 10.1161/STROKEAHA.118.021560

Dong, Y., Hassan, A., Zhang, Z., Huber, D., Dalageorgou, C., and Markus, H. S. (2003). Yield of screening for cadasil mutations in lacunar stroke and leukoaraiosis. Stroke 34, 203-205. doi: 10.1161/01.STR.0000048162.16852.88

Fazekas, F., Kleinert, R., Offenbacher, H., Payer, F., Schmidt, R., Kleinert, G., et al. (1991). The morphologic correlate of incidental punctate white matter hyperintensities on mr images. AJNR. Am. J. Neuroradiol. 12, 915-921.

Joutel, A., Corpechot, C., Ducros, A., Vahedi, K., Chabriat, H., Mouton, P., et al. (1996). Notch3 mutations in cadasil, a hereditary adult-onset condition causing stroke and dementia. Nature 383, 707-710. doi: 10.1038/383707a0

Joutel, A., Vahedi, K., Corpechot, C., Troesch, A., Chabriat, H., Vayssiere, C., et al. (1997). Strong clustering and stereotyped nature of notch 3 mutations in cadasil patients. Lancet 350, 1511-1515. doi: 10.1016/S0140-6736(97)08083-5

Kalaria, R. N., and Hase, Y. (2019). Neurovascular ageing and age-related diseases. Sub-Cellular Biochem. 91, 477-499. doi: 10.1007/978-981-13-3681-2_17

Kim, Y., Choi, E. J., Choi, C. G., Kim, G., Choi, J. H., Yoo, H. W., et al. (2006). Characteristics of cadasil in korea: a novel cysteine-sparing notch3 mutation. Neurology 66, 1511-1516. doi: 10.1212/01.wnl.0000216259.99811.50

Kim, Y. E., Yoon, C. W., Seo, S. W., Ki, C. S., Kim, Y. B., Kim, J. W., et al. (2014). Spectrum of notch3 mutations in korean patients with clinically suspicious cerebral autosomal dominant arteriopathy with subcortical infarcts and leukoencephalopathy. Neurobiol. Aging 35, 726.e721-726. doi: 10.1016/j.neurobiolaging.2013.09.004

Koizumi, T., Mizuta, I., Watanabe-Hosomi, A., Mukai, M., Hamano, A., Matsuura, J., et al. (2019). The cadasil scale-j, a modified scale to prioritize access to genetic testing for japanese cadasil-suspected patients. J. Stroke Cerebrovasc. Dis. 28, 1431-1439. doi: 10.1016/j.jstrokecerebrovasdis.2019.03.026

Lee, J. S., Ko, K., Oh, J. H., Park, J. H., Lee, H. K., Floriolli, D., et al. (2017). Cerebral microbleeds, hypertension, and intracerebral hemorrhage in cerebral autosomal-dominant arteriopathy with subcortical infarcts and leukoencephalopathy. Front. Neurol. 8:203. doi: 10.3389/fneur.2017.00203

Lee, Y. C., Chung, C. P., Chang, M. H., Wang, S. J., and Liao, Y. C. (2020). Notch3 cysteine-altering variant is an important risk factor for stroke in the taiwanese population. Neurology 94, e87-e96. doi: 10.1212/WNL.0000000000008700
Liu, X., Zuo, Y., Sun, W., Zhang, W., Lv, H., Huang, Y., et al. (2015). The genetic spectrum and the evaluation of cadasil screening scale in chinese patients with notch3 mutations. J. Neurol. Sci. 354, 63-69. doi: 10.1016/j.jns.2015.04.047

Matsushima, T., Conedera, S., Tanaka, R., Li, Y., Yoshino, H., Funayama, M., et al. (2017). Genotype-phenotype correlations of cysteine replacement in cadasil. Neurobiol. Aging 50, 169.e167-169.e114. doi: 10.1016/j.neurobiolaging.2016.10.026

Mizuno, T., Kondo, M., Ishigami, N., Tamura, A., Itsukage, M., Koizumi, H., et al. (2009). Cognitive impairment and cerebral hypoperfusion in a cadasil patient improved during administration of lomerizine. Clin. Neuropharmacol. 32, 113-116. doi: 10.1097/WNF.0b013e31816c82a6

Mizuno, T., Muranishi, M., Torugun, T., Tango, H., Nagakane, Y., Kudeken, T., et al. (2008). Two japanese cadasil families exhibiting notch3 mutation r75p not involving cysteine residue. Internal Med. 47, 2067-2072. doi: 10.2169/internalmedicine.47.1391

Mizuta, I., Watanabe-Hosomi, A., Koizumi, T., Mukai, M., Hamano, A., Tomii, Y., et al. (2017). New diagnostic criteria for cerebral autosomal dominant arteriopathy with subcortical infarcts and leukocencephalopathy in japan. J. Neurol. Sci. 381, 62-67. doi: 10.1016/j.jns.2017.08.009

Muino, E., Gallego-Fabrega, C., Cullell, N., Carrera, C., Torres, N., Krupinski, J., et al. (2017). Systematic review of cysteine-sparing notch 3 missense mutations in patients with clinical suspicion of cadasil. Int. J. Mol. Sci. 18, pii:E1964. doi: 10.3390/ijms18091964

Mukai, M., Mizuta, I., Watanabe-Hosomi, A., Koizumi, T., Matsuura, J., Hamano, A., et al. (2020). Genotype-phenotype correlations and effect of mutation location in japanese cadasil patients. J. Human Genetics. doi: 10.1038/s10038-020-0751-9. [Epub ahead of print].

Pantoni, L., Pescini, F., Nannucci, S., Sarti, C., Bianchi, S., Dotti, M. T., et al. (2010). Comparison of clinical, familial, and mri features of cadasil and notch3negative patients. Neurology 74, 57-63. doi: 10.1212/WNL.0b013e3181c7da7c

Pescini, F., Nannucci, S., Bertaccini, B., Salvadori, E., Bianchi, S., Ragno, M., et al. (2012). The cerebral autosomal-dominant arteriopathy with subcortical infarcts and leukoencephalopathy (cadasil) scale: a screening tool to select patients for notch3 gene analysis. Stroke 43, 2871-2876. doi: 10.1161/STROKEAHA.112.665927

Peters, N., Holtmannspotter, M., Opherk, C., Gschwendtner, A., Herzog, J., Samann, P., et al. (2006). Brain volume changes in cadasil: a serial mri study in pure subcortical ischemic vascular disease. Neurology 66, 1517-1522. doi: 10.1212/01.wnl.0000216271.96364.50

Regenhardt, R. W., Das, A. S., Lo, E. H., and Caplan, L. R. (2018). Advances in understanding the pathophysiology of lacunar stroke: a review. JAMA Neurol. 75, 1273-1281. doi: 10.1001/jamaneurol.2018.1073

Rutten, J. W., Dauwerse, H. G., Gravesteijn, G., van Belzen, M. J., van der Grond, J., Polke, J. M., et al. (2016). Archetypal notch3 mutations frequent in public exome: Implications for cadasil. Ann. Clin. Transl. Neurol. 3, 844-853. doi: $10.1002 / \operatorname{acn} 3.344$

Rutten, J. W., Haan, J., Terwindt, G. M., van Duinen, S. G., Boon, E. M. and Lesnik Oberstein, S. A. (2014). Interpretation of notch3 mutations in the diagnosis of cadasil. Expert Rev. Mol. Diagnostics 14, 593-603. doi: 10.1586/14737159.2014.922880

Santa, Y., Uyama, E., Chui, D. H., Arima, M., Kotorii, S., Takahashi, K., et al. (2003). Genetic, clinical and pathological studies of cadasil in japan: a partial contribution of notch 3 mutations and implications of smooth muscle cell degeneration for the pathogenesis. J. Neurol. Sci. 212, 79-84. doi: 10.1016/S0022-510X(03)00109-6

Sari, U. S., Kisabay, A., Batum, M., Tarhan, S., Dogan, N., Coskunoglu, A., et al. (2019). Cadasil with atypical clinical symptoms, magnetic resonance imaging, and novel mutations: two case reports and a review of the literature. J. Mol. Neurosci. 68, 529-538. doi: 10.1007/s12031-019-01313-Z

Singhal, S., Bevan, S., Barrick, T., Rich, P., and Markus, H. S. (2004). The influence of genetic and cardiovascular risk factors on the cadasil phenotype. Brain 127, 2031-2038. doi: 10.1093/brain/awh223

Singhal, S., Rich, P., and Markus, H. S. (2005). The spatial distribution of $\mathrm{mr}$ imaging abnormalities in cerebral autosomal dominant arteriopathy with subcortical infarcts and leukoencephalopathy and their relationship to age and clinical features. AJNR. Am. J. Neuroradiol. 26, 2481-2487.

Tan, R. Y. Y., Traylor, M., Megy, K., Duarte, D., Deevi, S. V. V., Shamardina, O., et al. (2019). How common are single gene mutations as a cause for 
lacunar stroke? A targeted gene panel study. Neurology 93, e2007-e2020. doi: 10.1212/WNL.0000000000008544

Tomimoto, H., Ohtani, R., Wakita, H., Lin, J. X., Ihara, M., Miki, Y., et al. (2006). Small artery dementia in japan: radiological differences between cadasil, leukoaraiosis and binswanger's disease. Dementia Geriatric Cognitive Disord. 21, 162-169. doi: 10.1159/000090677

Tournier-Lasserve, E., Joutel, A., Melki, J., Weissenbach, J., Lathrop, G. M., Chabriat, H., et al. (1993). Cerebral autosomal dominant arteriopathy with subcortical infarcts and leukoencephalopathy maps to chromosome 19q12. Nat. Genet. 3, 256-259. doi: 10.1038/ng0393-256

Ueda, A., Ueda, M., Nagatoshi, A., Hirano, T., Ito, T., Arai, N., et al. (2015). Genotypic and phenotypic spectrum of cadasil in japan: the experience at a referral center in kumamoto university from 1997 to 2014. J. Neurol. 262, 1828-1836. doi: 10.1007/s00415-015-7782-8

Veglio, F., Paglieri, C., Rabbia, F., Bisbocci, D., Bergui, M., and Cerrato, P. (2009). Hypertension and cerebrovascular damage. Atherosclerosis 205, 331-341. doi: 10.1016/j.atherosclerosis.2008.10.028

Viswanathan, A., Guichard, J. P., Gschwendtner, A., Buffon, F., Cumurcuic, R., Boutron, C., et al. (2006). Blood pressure and haemoglobin alc are associated with microhaemorrhage in cadasil: a two-centre cohort study. Brain 129, 2375-2383. doi: 10.1093/brain/awl177

Wang, Z., Yuan, Y., Zhang, W., Lv, H., Hong, D., Chen, B., et al. (2011). Notch 3 mutations and clinical features in 33 mainland chinese families with cadasil. J. Neurol. Neurosurgery Psychiatry 82, 534-539. doi: 10.1136/jnnp.2010. 209247
Watanabe, M., Adachi, Y., Jackson, M., Yamamoto-Watanabe, Y., Wakasaya, Y., Shirahama, I., et al. (2012). An unusual case of elderly-onset cerebral autosomal dominant arteriopathy with subcortical infarcts and leukoencephalopathy (cadasil) with multiple cerebrovascular risk factors. J. Stroke Cerebrovasc. Dis. 21, 143-145. doi: 10.1016/j.jstrokecerebrovasdis.2010.05.008

Wollenweber, F. A., Hanecker, P., Bayer-Karpinska, A., Malik, R., Bazner, H., Moreton, F., et al. (2015). Cysteine-sparing cadasil mutations in notch 3 show proaggregatory properties in vitro. Stroke 46, 786-792. doi: 10.1161/STROKEAHA.114.007472

Yamamoto, Y., Ihara, M., Tham, C., Low, R. W., Slade, J. Y., Moss, T., et al. (2009). Neuropathological correlates of temporal pole white matter hyperintensities in cadasil. Stroke 40, 2004-2011. doi: 10.1161/STROKEAHA.108.528299

Conflict of Interest: The authors declare that the research was conducted in the absence of any commercial or financial relationships that could be construed as a potential conflict of interest.

Copyright ( 2020 Okada, Washida, Irie, Saito, Noguchi, Tomita, Koga, Toyoda, Okazaki, Koizumi, Mizuta, Mizuno and Ihara. This is an open-access article distributed under the terms of the Creative Commons Attribution License (CC BY). The use, distribution or reproduction in other forums is permitted, provided the original author(s) and the copyright owner(s) are credited and that the original publication in this journal is cited, in accordance with accepted academic practice. No use, distribution or reproduction is permitted which does not comply with these terms. 